\title{
Deconstructing a path to reconstructing reproductive organs
}

\author{
David F. Albertini
}

Published online: 26 August 2011

(C) Springer Science+Business Media, LLC 2011

The scanning electron micrograph gracing our cover this month will prompt curiosity and ambiguity among our readership. While suggesting to some a landscape of otherworldly origins, and to others a spongiform likeliness you might find in a kitchen sink, it, in fact, portends the stuff of regenerative medicine that we are hearing more and more about these days. This image represents an artificial matrix used to culture pieces of ovary in an attempt to reconstruct and/or preserve the follicle reserve of ovarian tissue. Using such artificial substrates, studies from the laboratory of Dr. Ronit Abir, in Israel (Kedem et al., Scaffold for organ culture of cryopreserved-thawed human ovarian follicles), are among several published in the pages of JARG over the past year, signifying a course of research that has steadily been gaining momentum: gonad reconstruction.

The logistics behind such a monumental effort find their roots in the field of biomedical engineering, a staple of the regenerative medicine movement. And the momentum for such an endeavor has its origin in the emergent field of fertility preservation. In the case of the latter, the prospect of preserving ovarian or testicular function as a countermeasure to acquired or induced disorders such as premature ovarian failure, Turner's syndrome, or Sertoli cell-only syndrome has evoked two independent lines of research. On the one hand are those subscribing to the au naturel approach. Cryopreserving testis or ovary (or pieces thereof) harboring the elusive but much-sought-after germ cells is the goal here, with subsequent transplantation of these

Capsule Two divergent and distinct pathways are being pursued in ongoing efforts to build gonad equivalents suitable for the treatment of human infertility.

D. F. Albertini $(\bowtie)$

Kansas University Medical Center,

Kansas City, KS, USA

e-mail: dalbertini@kumc.edu tissues into donor patients. This approach has received encouragement, direction, and tractability from the pioneering studies of Silber; Gosden; Donnez and Meirow among others. The scenario exploited in these highly publicized cases involves whole ovary transplantation or transplantation of strips of ovarian cortex that have re-established cyclicity and reproductive competence to the small but fortunate number of patients who have benefitted from such treatments.

While many obstacles remain with respect to the use of tissue or whole organ transplantation, with respect to optimizing cryopreservation protocols, limiting damage from ischemia, and the genetic integrity of contained gametes, this au naturel approach attempts to maintain the invaluable supply of germ cells in a native environment or niche within which the subsequent steps of gametogenesis will proceed.

Yet another camp proffers a more ambitious agenda for preserving fertility, that I refer to as the "deconstructing reconstructionists". Let me explain. Accepting that our gonads are dualistic in function as both purveyors of the gametes upon which the next generation will derive and endocrine machines (and probably paracrine) in their secretory profile, not one, but two, functional attributes must be recapitulated in the design of artificial gonads. The plot immediately and necessarily thickens when one considers these as not so mutually exclusive operational endpoints for a self-respecting mammalian gonad. If you are designing a testis, then there is no choice but to establish an organ equivalent bearing both gameteproducing spermatogonial stem cells and the cellular and endocrine environments known to sustain the process of spermatogenesis. Thus, any facsimile of an artificial testis must embody a seminiferous tubule analog that satisfies the complex interaction between Sertoli, Leydig, and germ cells. Getting sperm out of tubule equivalents is another matter but, for now, we will assume that access to sperm 
from an appropriately temperature-regulated segment of male anatomy is plausible.

If you are designing an ovary, then matters of proximity to the Fallopian tube remain. This approach (de novo construction of an ovary equivalent) also implies anatomical proximity coupled to an ovulatory capacity. As we will see, such a feat may well exceed the expectations of the most optimistic among us. Instead, consider the first principles invoked by Carson and colleagues in their paper on an artificial ovary published in JARG last year (Krotz et al., J Assist Reprod Genet (2010) 27:743-750. DOI 10.1007/s10815-010-9468-6).

This group, working at Brown University, posits inclusion of the three principle cell types-oocytes, granulosa, and theca - that are reunited within or about a follicle-like fabricated mold. Evidence for cell viability after 72 hours is demonstrated, but the proof of the pudding rests in findings consistent with germ-cell viability and developmental competence for the enclosed oocytes. Stay tuned.

In contrast to building the functional unit of an ovary (or reconstructing a follicle from its basic cellular constituents, isolated from human ovaries at various stages of the menstrual cycle), a somewhat more pragmatic approach is also being pursued. In this case, pieces of ovary already containing the primordial follicle precursors of the finished product (Graafian follicle) are cultured upon a matrix like that illustrated on our cover this month. This alternative tact is being used by several laboratories and is founded on the principle that starting with a follicle constructed during ovarian biogenesis would increase the likelihood of sustaining it through the rigors of folliculogenesis. Perhaps, it is reasoned, a follicle and enclosed oocyte would take advantage of design principles imparted at birth. Call it a birthright strategy! Whatever you call it, and as with most studies aimed at sustaining follicle development in culture, assessments of functionality are typically biased in the direction of monitoring steroid production, fair and accurate measures of the steroidogenic health of the follicle.; however, whether this approach will prove useful for sustaining oocyte health and vitality AND an ability to generate healthy embryos after maturation and in vitro fertilization remains to be demonstrated. Rodent animal models for artificial ovaries capable of producing oocytes that generate live offspring are well established, but such endpoints have yet to be realized-either for human or subhuman primates.

Over and above the importance of offering patients whose fertility is or will be compromised through genetic, environmental and/or therapy-based factors, we should be mindful of the secretory roles our gonads play in everyday life, by providing a platform for the biogenesis of hormones and growth factors central to homeostasis, development, and general health.

In closing, it is befitting for those of us on the sidelines, eagerly awaiting future developments on gonadal replacement therapies, to encourage these laudable efforts in the two ways that seem most appropriate. First, keep abreast of these developments through the pages of JARG, as we continue to provide up-to-date coverage on matters of regenerative medicine that bear directly on our professional commitments to understand the fascinating intricacies of gonadal physiology. This knowledge base will color our dialogue with patients and colleagues during these exciting times in reproductive medicine. Second, cheer on these efforts as they continue to challenge the creativity, resourcefulness, and courage of our peers and their trainees, who have chosen to take on one of the most daunting problems of our time. Should you be in search of a way to vocally offer such support, might I boldly suggest using the cheer "GO-NADS", , taken from the annals of reproductive medicine over 30 years ago.

\footnotetext{
${ }^{1}$ Attribution to one Willian Moyle, then captain of the NADS softball team for the Laboratory for Human Reproduction and Reproductive Biology at Harvard Medical School in the early 1970s.
} 